\title{
Antinuclear antibodies in rosacea patients
}

\author{
Anna Woźniacka', Małgorzata Salamon¹, Daniel McCauliffe², Anna Sysa-Jędrzejowska ${ }^{1}$ \\ ${ }^{1}$ Department of Dermatology, Medical University of Lodz, Poland \\ Head: Prof. Anna Sysa-Jędrzejowska MD, PhD \\ 2Department of Dermatology, University of North Carolina at Chapel Hill; Private Practice, Rutland, VT USA \\ Head: Luis A. Diaz
}

Postep Derm Alergol 2013; XXX, 1: 1-5

DOI: $10.5114 /$ pdia.2013.33372

\begin{abstract}
Introduction: Rosacea is a common inflammatory disorder, characterized by a spectrum of facial manifestations. The clinical similarity to other dermatoses, like lupus erythematosus, might lead to misdiagnosis, particularly in patients with elevated antinuclear antibody titers.

Aim: To assess the frequency, titer and specificity of antinuclear antibodies in rosacea patients and correlate these findings with clinical features.

Material and methods: The study included 101 rosacea patients and 26 sex- and age-matched controls. Immunofluorescence antinuclear antibody testing was performed on HEp-2 substrates. Patients' sera with ANA titers of $1: 160$ or higher were evaluated by Euroline analysis.

Results: Over a half (53.5\%) of rosacea patients had an ANA titer greater than or equal to $1: 160$. Within this group $13.86 \%$ had a titer of $1: 320,8.91 \%$ had a titer of $1: 640$, and $6.93 \%$ had a titer of $1: 1,280$ or higher. The specificity of these antibodies could not be identified. Elevated ANA titers were present more often in women (55.8\%) than in men (44.15\%). Only two of 26 healthy volunteers had elevated ANA titers. One had a titer of $1: 160$ and the other of $1: 320$. During a two-year observation period, after the initial ANA testing, none of the patients with ANA titers above $1: 640$ developed an apparent autoimmune disorder.

Conclusions: Elevated ANA titers are commonly found in rosacea patients, what with simultaneously existing facial erythema and photosensitivity might lead to misdiagnosis of lupus erythematosus. Clinicians should beware of these findings to avoid misdiagnosing lupus erythematosus in rosacea patients with elevated ANA titers.
\end{abstract}

Key words: rosacea, antinuclear antibodies.

\section{Introduction}

Rosacea is a chronic inflammatory disorder with pathogenic mechanisms that are not fully understood. It affects mainly women in the third and fourth decade of their lives. According to the National Rosacea Society [1], disease recognition is based mainly on clinical criteria divided into primary and secondary categories. Primary criteria include: facial flushing (transient erythema), nontransient erythema, papules, pustules and telangiectases. The presence of one or more of these signs with a central facial distribution is considered highly indicative of rosacea. Secondary diagnostic criteria often appear with one or more of the primary features of rosacea. They include: burning or stinging skin sensations, elevated red plaques without epidermal changes, dry skin appearance, edema, ocular manifestations, peripheral location and phymatous changes.

There are four main rosacea subtypes: erythematelangiectatic, papulopustular, phymatous and ocular rosacea. Variants of rosacea, which do not strictly match the morphologic patterns or combinations as seen in these rosacea subtypes, may occur. The National Rosacea Society Expert Committee has recognized one such variant called granulomatous rosacea [1].

A diagnosis of rosacea is based on presenting clinical features as there are no specific histological findings or serologic markers. Hence, other conditions presenting with facial erythema, such as the butterfly rash of systemic lupus erythematosus (SLE) might cause diagnostic misinterpretation. Both diseases present more commonly in women and

Address for correspondence: Prof. Anna Woźniacka MD, PhD, Department of Dermatology, Medical University of Lodz, 5 Krzemieniecka Str., 94-115 Lodz, Poland, phone: +48 4268679 81, fax: +48 4268845 65, e-mail: wozniacka@bmp.net.pl Received: 8.08.2012, accepted: 13.11.2012. 
they intensify under the influence of solar radiation. Skin biopsy of the face is often avoided when possible to avoid facial scarring, thus making it more likely that a skin biopsy will not be performed that would help distinguish LE from rosacea. Physicians might otherwise rely on detecting autoantibodies by ANA screening to confirm a suspected diagnosis of LE-related facial erythema. It is therefore important to determine how frequently rosacea patients have elevated titers of autoantibodies by ANA testing.

\section{Aim}

This study aimed to determine the frequency, titer and specificity of antinuclear antibodies in rosacea patients and correlate these findings with age, sex, presence of autoimmune disease and clinical subset of rosacea.

\section{Material and methods}

The study was performed on 101 patients (77 women and 24 men) with rosacea and in 26 sex- and age-matched healthy individuals. The median age of rosacea patients was 50.4 years (range: 23-84 years). Each patient and control subject signed written informed consent before entry into the study that had been approved by the local Ethics Committee. All the subjects participating in our study underwent a thorough physical examination, and selected laboratory tests (total blood count, urinalysis, erythrocyte sedimentation rate (ESR), blood urea nitrogen (BUN), liver enzymes, serum creatinine, complement levels ( $\mathrm{C} 3, \mathrm{C} 4, \mathrm{CH} 50)$, and creatine kinase muscle enzyme levels). None of the enrolled patients showed signs or symptoms of SLE, other connective tissue disease or neoplasia. The diagnosis of rosacea was based on the criteria established by the National Rosacea Society Expert Committee on the Classification and Staging of Rosacea directions [1].

To detect serum autoantibodies, indirect immunofluorescence was perfomed on a neoplastic cell line established from a human laryngeal carcinoma (HEp-2) with the

Table 1. Titer of antinuclear antibodies in patients with rosacea and healthy controls

\begin{tabular}{lcccc}
\hline ANA titer & \multicolumn{2}{c}{$\begin{array}{c}\text { Patients with rosacea } \\
(n=101)\end{array}$} & \multicolumn{2}{c}{$\begin{array}{c}\text { Control group } \\
(n=26)\end{array}$} \\
\cline { 2 - 5 } & $n$ & $\%$ & $n$ & $\%$ \\
\hline Negative & 47 & 46.53 & 24 & 92.30 \\
\hline Positive & 54 & 53.46 & 2 & 7.70 \\
\hline $1: 160$ & 24 & 23.76 & 1 & 3.85 \\
\hline $1: 320$ & 14 & 13.86 & 1 & 3.85 \\
\hline $1: 640$ & 9 & 8.91 & - & - \\
\hline $1: 1,280$ & 4 & 3.96 & - & - \\
\hline $1: 2,560$ & 3 & 2.97 & - & - \\
\hline
\end{tabular}

standard kit from the EUROIMMUN company (Medizinische Labordiagnostika AG, Lubeck, Germany) according to the manufacturer's recommendations. A titer of $1: 160$ or higher was considered positive [2].

The assessment of the presence of circulating antibodies was made with the use of a fluorescence microscope. Sera that were positive at $1: 160$ or higher were tested for specific autoantibodies (RNP, Sm, Ro-60, Ro-52, La, PM-Scl, Jo-1, dsDNA, histone and ribosomal P-Protein) by the Euroline test (EUROIMMUN Medizinische Labordiagnostika AG, Lubeck, Germany). Patients who had elevated ANA titers were screened for other signs or symptoms of underlying autoimmune disease and ANA testing was repeated on these patients after 6 months and again after 2 years. A detailed medical history of the patient was obtained, including a review of symptoms, questions regarding the course and exacerbating factors of the rosacea, a medication history, and a history of sensitivity to sunlight.

\section{Results}

Fifty four of 101 rosacea patients (53.46\%) had an ANA titer of $1: 160$ or higher. Twenty-four patients $(23.76 \%)$ had the borderline ANA titer of $1: 160$. Titers of $1: 320$ and $1: 640$ were present in $14(13.86 \%)$ and 9 (8.91\%) patients, respectively. ANA titers of $1: 1,280$ to $1: 2,560$ were present in 7 patients $(6.93 \%)$. In the control group, ANA titers of $1: 160$ and $1: 320$ were observed in 1 person each, out of 26 (7.68\%) patients. The results are illustrated in Table 1.

Thirteen patients under 40 years of age had elevated ANA titers out of 23 patients (56\%). Thirty-one patients aged between 40 and 60 had elevated titers out of 58 patients (53.4\%) and in the age group over 60 years, elevated ANA titers were found in 10 out of 20 patients (50\%). Statistical analysis did not reveal any correlations between the patients' age and the ANA titers. A detailed analysis of ANA titers is shown in Figure 1.

A higher frequency of elevated ANA titers was observed in female rosacea patients (43 out of $77(55.8 \%)$ ) than in

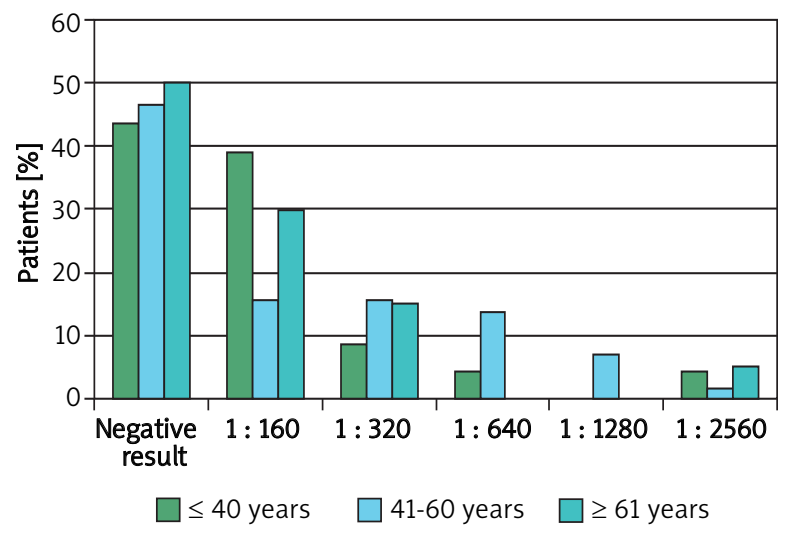

Figure 1. Distribution of antinuclear antibody presence among patients with rosacea in the age ranges 
Table 2. Relationship between presence of antinuclear antibodies and clinical form of rosacea

\begin{tabular}{lccccccc}
\hline Clinical form & $\begin{array}{c}\text { Absence } \\
\text { of ANA }\end{array}$ & \multicolumn{3}{c}{ ANA titer } & & & $\begin{array}{c}\text { Presence } \\
\text { of ANA }\end{array}$ \\
\cline { 2 - 7 } & & $1: 160$ & $1: 320$ & $1: 640$ & $1: 1,280$ & $1: 2,560$ & \\
\hline ETR $(n=35)$ & $18(17.8 \%)$ & $9(8.9 \%)$ & $3(3.0 \%)$ & $2(2.0 \%)$ & $2(2.0 \%)$ & $1(1.0 \%)$ & $17(16.8 \%)$ \\
\hline PPR $(n=57)$ & $24(23.8 \%)$ & $13(12.9 \%)$ & $10(9.9 \%)$ & $6(5.9 \%)$ & $2(2.0 \%)$ & $2(2.0 \%)$ & $33(32.7 \%)$ \\
\hline Other $(n=9)$ & $5(5.0 \%)$ & $2(2.0 \%)$ & $1(1.0 \%)$ & $1(1.0 \%)$ & $0(0.0 \%)$ & $0(0.00 \%)$ & $4(4.0 \%)$ \\
\hline
\end{tabular}

ETR - erythematotelangiectatic rosacea, PPR - papulopustular rosacea

the male group (11 out of $24(45.8 \%))$. The differences were not statistically significant, however $(p=0.39)$.

In most patients, a speckled or granular pattern of nuclear fluorescence was observed. In a few cases, a homogenous nuclear, mitochondrial or cytoplasmic type of fluorescence was detected.

None of rosacea patients or the control group had specific autoantibodies detected by the Euroline test (RNP, Sm, Ro-60, Ro-52, La, PM-Scl, Jo-1, dsDNA, histone and ribosomal P-Protein).

Thirty-five patients in the study had the erythematous subset with telangiectasia, 57 had the papulopustular variant, 6 had the hypertrophic variant and 3 had the ocular type. Due to the small number of patients with the hypertrophic and ocular subsets, the comparative analysis was performed only on the patients with the erythematous with telangiectasia, and papulopustular subsets. No correlation between ANA titer and clinical rosacea subset was observed $(p>0.05)$. The results are shown in Table 2. In the examined group, $25 \%$ of patients notified mild symptoms of coronary artery disease (chest pain, shortness of breath, fast heartbeat, weakness, dizziness, and (or) increased sweating during physical activity). Within this group, $45.5 \%$ had antinuclear antibodies at a titer greater than or equal to $1: 160$.

A repeat ANA titer 6 months after the first revealed a reduction or increase of only one dilution, which is within the variance limit of the method employed. During a 2-year clinical observation, none of the patients with an ANA titer above $1: 640$ developed an autoimmune disease. In 26 healthy volunteers, only 2 had elevated ANA titers. In one, the titer was $1: 160$ and in the other, it was $1: 320$.

\section{Discussion}

It is well established that in the course of many autoimmune diseases, antibodies arise that react with antigens in the patient's own cells. Although in some autoimmune diseases, such as LE, antibodies may have a diagnostic value, many autoantibodies are not disease specific [3]. Antinuclear antibodies are one of the diagnostic criteria of SLE, but only dsDNA and Sm are regarded as specific disease markers. However, in many patients with high ANA titers the antibody specificity is unknown [4].
Indirect immunofluorescence testing is considered to be a sensitive method of detecting antinuclear antibodies (ANA) in which the character of fluorescence depends on the type of antibodies present. There are several types of immunofluorescence patterns. They include a homogenous type (from antibodies targeting DNA and histones), a particulate type (produced by RNP, Ro/SS-A, La/SS-B antibodies), a ring pattern (from DNA antibodies), a nucleolar pattern (from nucleolar antigens such as PM-Scl), and a granular pattern (from Sm, U1RNP, Mi-2 antibodies) [4].

Many different antinuclear antibodies have been identified, however their pathogenic role in the development of diseases still remains controversial. Generally accepted is the etiological role of dsDNA in the development of lupus nephritis, anti-Ro/SS-A in the development of changes in the skin and cardiac conducting system in neonatal lupus erythematosus, and possibly anti-U1RNP in mixed connective tissue disease [4].

Worth mentioning is the fact that as newer more sensitive autoantibody detection assays have been developed, such as the use of cultured human cell substrates, the presence of autoantibodies are more often detected in healthy people. Studies performed on a large numbers of normal people confirmed that the presence of antibodies at low titers (less than $1: 160$ ) does not have a significant diagnostic value [2]. Low ANA titers are present more often in women than in men and the frequency of that phenomenon increases with age [5]. Anderson [6] found the presence of ANA titers in the range of $1: 10-1: 80$ in 2\% of adults between 21 to 40 years of age, in $9 \%$ between 41 to 60 years of age, and in $25 \%$ over 65 years of age. Shu et al. [7] found low ANA titers in most healthy adults between 18 and 66 years of age. Titers of $1: 10$ to $1: 20$ were present in $45 \%$ of examined persons, a titer of $1: 40$ in $19 \%$ and a titer of $1: 80$ in $5.6 \%$. At present, it is generally accepted that the presence of fluorescence on cultured human cell substrates, by ANA testing, at a $1: 160$ dilution (titer), is likely to be a significant, or positive result. Lower titers are generally regarded as negative [8].

The literature data indicate that there are many disorders associated with the development of autoantibodies and there are numerous reports of drug-induced ANAs [9]. None of the rosacea patients in this study took medications known to induce autoantibodies. Some authors 
imply that antinuclear antibodies are present more often in old patients and explain that it is due to the development of atherosclerotic changes in blood vessels. Grainger and Bethell revealed that the presence of ANA, commonly associated with autoimmune diseases, is substantially more prevalent among subjects with severe coronary atherosclerosis than those with normal coronary arteries. This association merits further assessment as a potentially useful indicator of an increased risk of coronary heart disease. Within the comparable control group in this rosacea study, ANAs were found only in $17 \%$ of patients [10]. Brusca et al. revealed the presence of ANA at titers between $1: 40$ and $1: 640$ in $32 \%$ of patients with myocardial infarction, transient ischemia or ischemic cerebral stroke, the diseases in which atherosclerosis is regarded as a main etiopathological factor [11].

In our study, $25 \%$ of analyzed patients noticed mild symptoms suggestive of possible coronary artery disease (chest pain, shortness of breath, fast heartbeat, weakness, dizziness, and (or) increased sweating during physical activity) and $45.5 \%$ of these patients had ANA titers greater than or equal to $1: 160$. Further details regarding the development of cardiac disease in these patients since the study began, have not yet been analyzed.

Imran's group examined patients with neoplastic disease and showed the presence of ANA, at $1: 100$ serum dilution (on the less sensitive goat kidney substrate), in $40 \%$ of patients [12]. Neoplastic changes in cells seem to stimulate the development of autoantibodies in some instances. The authors stress however that autoantibody titers found in cancer patients are usually present at lower titers than found in autoimmune disorders, like SLE. Autoantibodies are also more often present in patients with an infectious disease such as mononucleosis, malaria, tuberculosis and chronic hepatitis [8].

Among the rosacea patients in this study, there were no signs of an underlying neoplastic or infectious disease. In 13 patients with elevated antibodies titers of $1: 640$, who were evaluated 2 years later, none had developed cancer.

Ethnic differences may influence the frequency of the antinuclear antibodies in healthy people. Studies performed on blood donors showed ethnic discrepancies in the frequency of positive results. Within a population from Saudi Arabia, ANAs were detected in $4.2 \%$ of patients (by indirect immunofluorescence (IIF), at a titer of 1 : 20 on HEp-2 cells) [13], from Belgium in 13\% (IIF, 1 : 40, HEp-2) [14], from Mexico in $4.7 \%$ of patients (IIF, $1: 40$, HEp-2) [15], from Canada in 15.9\% (IIF, $1: 40$, HEp-2) [16], from Sweden in 8.2\% (IIF, $1: 100, \mathrm{HEp}-2$ ) [2], and from Poland in 12\% (by EIA) [18]. Unfortunately, these studies considered titers less than $1: 160$ as positive, so these frequencies are likely to be of little significance, since the majority of the ANA testing was performed on human cultured cell lines (HEp-2 cells) that detect autoantibodies at low titers even in normal individuals. The Hayashi study performed ANA testing on 2,181 Japanese patients and found antinuclear antibodies in low titers of 1 : 40 in $25.9 \%$ and at 1 : 160 dilution in $9.5 \%$ of healthy people with a female predominance [19]. Moreover, among 566 clinically healthy individuals with an ANA titer of $1: 40$ or higher, disease-specific ANAs, like Ro/SS-A, U1RNP and anti-centromere antibodies were found in 100 of the 566 individuals. In 60 of the 100 patients who were available, 30\% (18/60) actually manifested systemic rheumatic diseases, while $50 \%$ showed no detectable signs or symptoms of rheumatic diseases. Despite the fact that within our group of rosacea patients, antinuclear antibodies were present in a great number of patients, the specificity of these antibodies were not identified, and none of our rosacea patients had an apparent autoimmune disease.

Skin lesions in rosacea, especially in the beginning of the disease, are characterized by facial flushing with telangiectasia. Rosacea is more common in women and can be exacerbated by sun exposure. A similar clinical picture is observed in the course of different morphological subsets of LE. Since the above described features are present in the course of both diseases (LE and rosacea) and affect adult persons, the coincidental occurrence of other ailments such as arthritis, might allow four diagnostic criteria of SLE to be fulfilled (malar erythema, photosensitivity, positive ANA). Such rosacea patients might thus be misdiagnosed as SLE. Black et al. highlight diagnostic problems in differentiation between LE and rosacea [8]. Out of 21 patients misdiagnosed with LE in a rheumatic skin disease clinic, 16 of the misdiagnosed patients had rosacea. Five of these patients had an ANA titer equal to or higher than $1: 160$, but on repeat testing (on HEp-2 cells) all had titers less than or equal to $1: 40$ [8]. Similarly, Sontheimer underlined the diagnostic problems between distinguishing those two diseases, especially when patients are ANA positive [20].

Our results revealed a higher frequency of antinuclear antibodies in rosacea patients, compared to an earlier Greek study that found 19 of 90 (21.1\%) rosacea patients with antinuclear antibodies titers that fluctuated from $1: 40$ to $1: 160$, on HEp-2 cells [21]. Why we found a larger percentage of rosacea patients with elevated ANA titers compared to this study and that of Black et al. [8] is uncertain. All three studies utilized the sensitive cultured human HEp-2 cells as the substrate for ANA testing. The lack of differences in the ANA titers in our rosacea patients 6 months later indicate that the elevated ANA titers were not spuriously elevated. Additional ANA studies in rosacea patients should be done to address this discrepancy.

A prospective study performed on children and youths with no clinical symptoms of autoimmune disease but with the presence of low titer antinuclear antibodies $(1: 20-1: 80$ on HEp-2 cells) revealed no predilection for the development of an autoimmune disease, over a 13-month to 3-year period [16, 22, 23].

Arbucle's group evaluated stored blood samples from 130 people that were collected years before these patients were diagnosed with SLE. Among this group, 78\% had detectable antibodies by ANA at a titer of $1: 120$ or high- 
er (up to 9.4 years earlier; mean: 3.3 years) [24]. The authors conclude that autoantibodies are typically present many years before the diagnosis of SLE. However, specific autoantibodies appear closer to the onset of SLE.

In our study, none of the 13 rosacea patients with antinuclear antibodies at titers equal to or higher than $1: 640$ developed signs of autoimmune disease during a two-year observation period. However, it seems advisable to follow patients with high titers of antinuclear antibodies for the development of autoimmune diseases, as suggested by Perilloux et al. [25].

\section{Conclusions}

Clinicians should be aware of our findings that elevated ANA titers are not infrequently found in rosacea patients. Thus, less significance should be given to an elevated ANA titer when trying to distinguish LE from rosacea. In some cases specific antibody tests (e.g., Ro/SS-A, dsDNA, Sm) or a skin biopsy might be helpful to distinguish between these two disorders with overlapping cutaneous features. Although in a 2-year observation period none of our rosacea patients with elevated ANA titers developed an autoimmune disorder, it would be prudent to follow such patients, over time, to look for signs or symptoms of a developing autoimmune disorder.

\section{Acknowledgments}

This work was supported by grant no. 503/1-15201/503-01 from the Medical University of Lodz.

\section{References}

1. Wilkin J, Dahl M, Detmar M, et al. Standard classification of rosacea: Report of the National Rosacea Society Expert Committee on the Classification and Staging of Rosacea. J Am Acad Dermatol 2002; 46: 584-9.

2. Tan EM, Feltkamp TE, Smolen JS, et al. Range of antinuclear antibodies in "healthy" individuals. Arthritis Rheum 1997; 40: 1601-11.

3. Egner W. The use of laboratory tests in the diagnosis of SLE. J Clin Pathol 2000; 53: 424-32.

4. Smeenk RJ. Antinuclear antibodies: cause of disease or caused by disease? Rheumatology 2000; 39: 581-4.

5. Lipscomb MF, Cope LD, Stephens GL, et al. Comparison of substrates for the detection of antinuclear antibodies in normals and in patients with connective tissue and other diseases. Diag Immunol 1984; 2: 181-7.

6. Anderson P. Correlation of smooth-muscle and nuclear antibodies in normal subjects. Clin Exp Immunol 1977; 27: 74-7.

7. Shu S, Nisengard RJ, Hale WL, Beutner EH. Incidence and titers of antinuclear, antismooth muscle, and other autoantibodies in blood donors. J Lab Clin Med 1975; 86: 259-65.

8. Black AA, McCauliffe DP, Sontheimer R. Prevalence of acne rosacea in a rheumatic skin disease subspecialty clinic. Lupus 1992; 1: 229-37.

9. Jiménez-Alonso J, Jaimez L, Sabio JM, et al. Atorvastatin-induced reversible positive antinuclear antibodies. Am J Med 2002; 112: 329-30.
10. Grainger DJ, Bethell HW. High titers of serum antinuclear antibodies, mostly directed against nucleolar antigens, are associated with the presence of coronary atherosclerosis. Ann Rheum Dis 2002; 61: 97-9.

11. Brusca I, Sarullo F, Fazio M, Grainger D. Presence of antinuclear antibodies and coronary heart disease. Ann Rheum Dis 2002; 61: 1038-9.

12. Imran A, Neelam F, Tariq M. Incidence of circulating antinuclear antibodies in cancer patients. Indian J Med Sci 2003; 57: 113-6.

13. Baig MM, Shere SJ. Prevalence of autoantibodies in Saudi population. J Med 1989; 20: 286-90.

14. de Vlam K, De Keyser F, Verbruggen G, et al. Detection and identification of antinuclear autoantibodies in the serum of normal blood donors. Clin Exp Rheumatol 1993; 11: 393-7.

15. Vazquez-Del Mercado M, Gonzalez-Bernaldez M, Garcia-De La Torre I. Prevalence of antinuclear antibodies in a Huichol population of Mexico. Lupus 1995; 4: 164-5.

16. Hilário MO, Len CA, Roja SC, et al. Frequency of antinuclear antibodies in healthy children and adolescents. Clin Pediatr 2004; 46: 637-4.

17. Tan M, Feltkamp T, Smolen J, et al. Range of antinuclear antibodies in "healthy" individuals. Arth Rheum 1997; 40: 1601-11.

18. Śpiewak R, Stojek N. Presence of antinuclear antibodies among Eastern-Polish rural inhabitants. Ann Agric Environ Med 2003; 10: 207-9.

19. Hayashi N, Koshiba M, Nishimura K. Prevalence of diseasespecific antinuclear antibodies in general population: estimates from annual physical examinations of residents of a small town over a 5-year period. Mod Rheumatol 2008; 18: 153-60.

20. Sontheimer RD. A young woman with a photosensitive butterfly facial rash, fatigue, anemia, and positive antinuclear antibody. Arthritis Rheum 1993; 36: 871-4.

21. Lazaridou E, Apalla Z, Sotiraki S, et al. Clinical and laboratory study of rosacea in northern Greece. JEADV 2010; 24: 410-4.

22. Cabral DA, Petty RE, Fung M, Malleson PN. Persistent antinuclear antibodies in children without identifiable inflammatory rheumatic or autoimmune disease. Pediatrics 1992; 89: 441-4.

23. Deane PM, Liard G, Siegel DM, Baum J. The outcome of children referred to a pediatric rheumatology clinic with a positive antinuclear antibody test but without an autoimmune disease. Pediatrics 1995; 95: 892-5.

24. Arbuckle MR, McClain MT, Rubertone MV, et al. Development of autoantibodies before the clinical onset of systemic lupus erythematosus. N Engl J Med 2003; 349: 1526-33.

25. Perilloux BC, Shetty AK, Leiva LE, Gedalia A. Antinuclear antibody (ANA) and ANA profile tests in children with autoimmune disorders: a retrospective study. Clin Rheumatol 2000; 19: 200-3. 